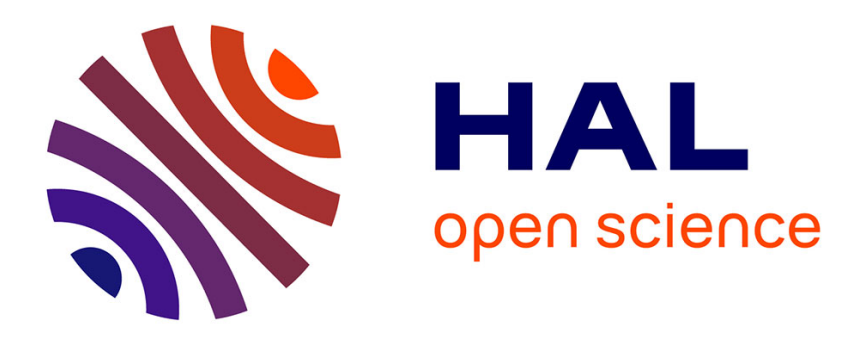

\title{
LA MULTIBANCARITÉ DES ENTREPRISES
}

Catherine Refait-Alexandre

\section{To cite this version:}

Catherine Refait-Alexandre. LA MULTIBANCARITÉ DES ENTREPRISES. Revue Economique, 2003, 54 (3), pp.649 à 661. 10.3917/reco.543.0649 . hal-01391629

\section{HAL Id: hal-01391629 \\ https://hal.science/hal-01391629}

Submitted on 3 Nov 2016

HAL is a multi-disciplinary open access archive for the deposit and dissemination of scientific research documents, whether they are published or not. The documents may come from teaching and research institutions in France or abroad, or from public or private research centers.
L'archive ouverte pluridisciplinaire HAL, est destinée au dépôt et à la diffusion de documents scientifiques de niveau recherche, publiés ou non, émanant des établissements d'enseignement et de recherche français ou étrangers, des laboratoires publics ou privés. 


\section{LA MULTIBANCARITÉ DES ENTREPRISES \\ Choix du nombre de banques et choix du nombre de banques principales \\ Catherine Refait-Alexandre}

Presses de Sciences Po (P.F.N.S.P.) | « Revue économique »

2003/3 Vol. 54 | pages 649 à 661

ISSN 0035-2764

ISBN 2724629515

Article disponible en ligne à l'adresse :

http://www.cairn.info/revue-economique-2003-3-page-649.htm

\section{Pour citer cet article :}

Catherine Refait-Alexandre, « La multibancarité des entreprises. Choix du nombre de banques et choix du nombre de banques principales », Revue économique 2003/3 (Vol. 54), p. 649-661.

DOI 10.3917/reco.543.0649

Distribution électronique Cairn.info pour Presses de Sciences Po (P.F.N.S.P.).

(C) Presses de Sciences Po (P.F.N.S.P.). Tous droits réservés pour tous pays.

La reproduction ou représentation de cet article, notamment par photocopie, n'est autorisée que dans les limites des conditions générales d'utilisation du site ou, le cas échéant, des conditions générales de la licence souscrite par votre établissement. Toute autre reproduction ou représentation, en tout ou partie, sous quelque forme et de quelque manière que ce soit, est interdite sauf accord préalable et écrit de l'éditeur, en dehors des cas prévus par la législation en vigueur en France. Il est précisé que son stockage dans une base de données est également interdit. 


\title{
La multibancarité des entreprises
}

\author{
Choix du nombre de banques \\ et choix du nombre de banques principales
}

\author{
Catherine Refait*
}

\begin{abstract}
Nous vérifions empiriquement l'impact de la qualité d'une entreprise sur le nombre et la concentration des banques qui la financent en nous fondant sur les conclusions de la littérature théorique. Nous utilisons un échantillon de PME industrielles françaises pour lesquelles nous disposons de données comptables et d'informations relatives à l'octroi de prêts bancaires. Le nombre total de banques auprès desquelles les firmes s'endettent apparaît d'autant plus élevé que la qualité des entreprises est faible, de manière à échapper à une surveillance trop étroite. En revanche, la qualité des entreprises ne semble que peu liée, et négativement, avec leur stratégie de nouer une relation privilégiée avec plusieurs banques, stratégie supposée permettre de ne pas subir de capture informationnelle.
\end{abstract}

\section{MULTIPLE BANKING RELATIONSHIPS: CHOICE OF THE NUMBER OF MAIN BANKS AND CHOICE OF THE NUMBER OF BANKS}

This empirical paper focuses on the total number of banks and the number of main banks (banks that play an important and durable role in the financing) of firms. We test two major results of the theoretical literature: Low quality firms are likely to choose a high number of banks, in order to escape from a close monitoring. Strong multiple banking relationships benefit high quality firms, for they don't pay the informational rent that a single bank would have extracted. We use accounting variables and credit declarations for industrial French firms. We show that the total number of banks increases when the economic profitability of the firm decreases. No important link appears between the performance of the firms and their number of main banks.

Classification JEL : G32, D82, C10

La relation banque-entreprise a évolué vers une plus grande multibancarité tant aux États-Unis qu'en Europe, depuis l'intensification de la concurrence sur le marché bancaire au milieu des années 1980. En France par exemple, les PME nouent en moyenne des relations avec trois banques (Actualité bancaire [2001]). Cet état de fait a suscité de nouvelles interrogations théoriques : la multibancarité

* Université Paris IX Dauphine. CERPEM-CREFED, Place Maréchal-de-Lattre-de-Tassigny 75775 Paris Cedex 16. E-mail : catherine.refait@dauphine.fr

Merci à l'Observatoire des entreprises de la Banque de France grâce auquel cette étude a pu être réalisée. Je remercie Mireille Bardos ainsi que Frédéric Lobez et Nicolas Eber pour leurs remarques. Je demeure seule responsable du contenu de cet article. 
est-elle uniquement le résultat d'une stratégie des banques pour conquérir des parts de marché? Est-elle un phénomène parfaitement exogène, dû au simple fait que les crédits sont d'un montant trop élevé pour être pris en charge par une banque seule ( $c f$. Winton [1995])? La multibancarité n'est-elle pas, au contraire, une décision émanant des entreprises? Dès lors, comment déterminent-elles le nombre des banques auprès desquelles elles s'endettent? Une autre question se pose : pourquoi certaines entreprises nouent-elles des relations privilégiées avec plusieurs banques? Nous appellerons ces banques des «banques principales », c'est-à-dire des banques qui représentent une part importante dans l'endettement global de l'entreprise et avec lesquelles l'entreprise entretient des relations durables. Si la question du nombre de banques rencontre de plus en plus de réponses théoriques et empiriques, la question de la concentration du financement bancaire reste en suspens.

Une première forme de réponse à ces interrogations a été apportée par les modèles relevant de la théorie bancaire des années 1980 (Diamond [1984], Fama [1985]). Une relation étroite entre l'entreprise et sa banque incite cette dernière à réaliser un investissement informationnel important. Une entreprise de qualité bénéficie alors notamment d'un accès au capital plus aisé et d'un soutien financier en cas de crise financière plus fréquent. La multibancarité peut s'expliquer par une stratégie d'entreprises de qualité médiocre, pour qui la dilution de l'information et l'assouplissement de la surveillance du créancier sont favorables. La stratégie des entreprises de disposer de plusieurs banques « principales » est différente. Une de ses raisons d'être peut être trouvée au sein des différents modèles relatifs aux incidences préjudiciables d'une relation bancaire exclusive, mises en avant initialement par Sharpe [1990]. Disposer de plusieurs banques permet aux entreprises de ne pas supporter la rente informationnelle d'un créancier unique, puisqu'une banque est supposée disposer d'informations privilégiées par rapport aux autres créanciers. Les entreprises de bonne qualité ont donc intérêt à nouer des relations privilégiées avec plusieurs banques.

Or il nous semble qu'une banque qui ne joue qu'un faible rôle dans le financement de l'entreprise est peu incitée à investir informationnellement; son rôle n'est donc pas conforme à celui prédit par la théorie. Nous désirons alors faire une distinction entre le choix du nombre total de banques et le choix du nombre de banques représentant une part importante dans l'endettement de l'entreprise. Nous nous proposons de vérifier empiriquement l'existence de ces deux stratégies différentes à partir de données individuelles de firmes françaises, afin de mettre en avant que la qualité des entreprises est liée négativement avec leur nombre total de banques mais qu'elle est liée positivement avec leur nombre de banques principales.

Les études empiriques relatives aux causes de la multibancarité ne font pas la distinction entre nombre total de banques et nombre de banques susceptibles d'être informées; elles ne considèrent que le premier ou considèrent qu'une seule banque peut être qualifiée de «principale» (De Bodt et al. [2001]). Detragiache et al. [2000] montrent que la profitabilité des entreprises influence positivement le choix d'une relation multibancaire plutôt qu'une relation exclusive. En revanche, il n'existe pas de lien significatif entre le nombre total de banques et la profitabilité des entreprises. Les résultats de Ongena et Smith [2001] vont dans le même sens : le fait que les entreprises multibancarisées se caractérisent par des relations bancaires de moins longue durée que les autres tendrait à corroborer l'idée qu'elles sont moins captives. Foglia et al. [1998] mettent en évidence une 
relation positive entre la fragilité des entreprises et leur nombre de banques. D'autres études existent, mettant en exergue des éléments explicatifs différents : Berger et al. [2001] montrent que les petites entreprises ont tendance à s'endetter auprès d'une banque unique, d'après les auteurs à cause du coût élevé de la recherche d'informations les concernant. Ils mettent également en évidence que les entreprises anticipant un risque de faillite de leur banque désirent se multibancariser. De Bodt et al. [2001] s'interrogent sur l'avantage que pourraient tirer les petites entreprises d'une relation multibancaire afin d'éviter un rationnement du crédit. Des stratégies variées apparaissent : les très petites entreprises liées à des banques de taille élevée ont intérêt à ne pas concentrer leur financement à cause du coût prohibitif de l'investissement informationnel alors que les plus grandes entreprises ont au contraire intérêt à avoir un nombre de banques faible. Enfin, citons Ongena et Smith [2000], qui montrent que les différences institutionnelles et légales entre les pays expliquent les divergences européennes en matière de multibancarité.

Notre étude empirique veut, au contraire, mettre l'accent sur le rôle informationnel différent des banques selon leur implication dans le financement de l'entreprise. L'étude porte sur des entreprises industrielles françaises, pour lesquelles nous disposons de données de la Centrale des risques de la Banque de France. Pour chaque entreprise, nous connaissons le cumul de l'ensemble des crédits (d'un encours supérieur à 700000 F) octroyés semestriellement par chacune de ses banques, sur la période 1993-1997. Les données ne présentent donc pas un caractère exhaustif. Elles constituent néanmoins une information rarement disponible sur le financement bancaire des entreprises. Nous disposons aussi de données de la Centrale des bilans de la Banque de France : bilans et comptes de résultat issus des déclarations fiscales des entreprises, pour la période 1993-1997 également.

La première section expose les principaux résultats de la littérature théorique relative au choix du nombre de banques. Nous dégageons alors les deux hypothèses que nous nous proposons de tester : l'impact de la qualité des entreprises sur le nombre total de banques et sur la stratégie de banque principale multiple. Nous recensons ensuite les autres facteurs susceptibles d'intervenir dans la détermination du nombre de banques et du nombre de banques principales. La deuxième section décrit les données utilisées : elle présente les caractéristiques générales des entreprises considérées, puis propose une description statistique de leur multibancarité et enfin spécifie les variables expliquées et les variables explicatives retenues. La méthodologie économétrique choisie et les résultats empiriques font l'objet de la troisième section.

\section{COMMENT L'ENTREPRISE DÉTERMINE-T-ELLE LA NATURE DE SES RELATIONS BANCAIRES?}

L'objet de cette section est de présenter les modèles relatifs aux avantages et inconvénients pour l'entreprise de la multibancarité afin de dégager les hypothèses que nous nous proposons de tester. Nous présentons d'abord les modèles fondés sur l'idée qu'une relation exclusive est bénéfique aux entreprises de qualité, puis nous évoquons les modèles qui, au contraire, envisagent les inci- 
dences négatives d'une telle relation. Enfin, nous recensons les autres éléments susceptibles d'expliquer le choix par l'entreprise de ses relations bancaires.

\section{Avantages d'une relation banque-entreprise exclusive et lien entre qualité de l'entreprise et nombre total de ses banques}

La théorie bancaire développée dans les années 1980 (Diamond [1984], Fama [1985] notamment) a mis en avant les raisons pour lesquelles les entreprises recourent au marché bancaire pour se financer. Elle a ainsi fondé la spécificité de la banque sur l'avantage informationnel dont elle dispose par rapport aux autres créanciers. Cet avantage est supposé accru par l'établissement d'une relation intense et durable avec l'entreprise, notamment grâce aux économies d'échelle permises ${ }^{1}$. Une relation privilégiée banque-entreprise permet donc une bonne connaissance du débiteur par son créancier ainsi qu'une confiance entre les partenaires. L'entreprise se voit alors offrir un accès au capital plus aisé $(c f$., par exemple, les études empiriques de De Bodt et al. [2000], Agarwal et Elston [2001]) et des taux d'intérêt plus faibles ( $c f$. notamment les études empiriques de D'auria et al. [1999] et de Vaubourg [2000]).

A contrario, une relation bancaire moins concentrée réduit l'incitation de la banque principale à réaliser un investissement pour recueillir des informations et contrôler l'entreprise ( $c f$. notamment Carletti [2002]). L'encours des crédits qu'elle octroie annuellement peut ne plus être suffisant pour justifier de telles dépenses. De plus, la banque ne dispose plus de l'engagement tacite de l'entreprise que la relation sera durable, ce qui permettait également la rentabilisation de l'investissement informationnel. Une entreprise de mauvaise qualité - ou qui anticipe une détérioration de sa performance économique - a donc intérêt à se multibancariser pour échapper au contrôle strict d'une banque principale fortement impliquée dans son financement. Cette proposition constitue la première hypothèse que nous désirons tester :

HYPOTHÈSE $\mathrm{n}^{\circ} 1$. Une entreprise de mauvaise qualité ou anticipant une dégradation de sa qualité se caractérise par un nombre total de banques élevé.

\section{Inconvénients d'une relation banque-entreprise exclusive et lien entre qualité de l'entreprise et nombre total de ses banques principales}

Sharpe [1990] puis Rajan [1992] ont mis en évidence que l'information privée détenue par la banque lui confère un monopole de fait. L'entreprise est alors captée car les autres créanciers lui proposent des conditions moins favorables; la banque extrait l'intégralité de sa rente informationnelle et l'entreprise n'est pas incitée à fournir des efforts importants pour mener à bien ses projets d'investis-

1. La banque tire en partie son avantage informationnel de l'économie d'une duplication inutile des coûts de monitoring (Diamond [1984]). Ce phénomène est d'autant plus marqué que le montant des crédits octroyés par la banque à l'entreprise est élevé. 
sement. Au contraire, tisser des liens privilégiés avec plusieurs banques permet aux entreprises d'échapper à cette situation. L'incitation de l'entreprise à réaliser des efforts est alors rétablie et la situation est optimale (Von Thadden [1994]). Seules deux banques «principales » sont néanmoins nécessaires pour rétablir l'optimalité. Afin d'expliquer pourquoi certaines entreprises nouent des liens étroits avec plus de deux banques, Detragiache et al. [2000] mettent en avant le risque qu'une banque puisse, de façon momentanée, ne pas financer l'entreprise. Si celle-ci dispose d'une seule banque principale, aucune autre banque n'acceptera de la financer pour des problèmes de sélections adverses ( $c f$. Sharpe [1990]). Disposer de plusieurs banques principales, donc informées, permet de réduire ce risque : en cas d'illiquidité temporaire de l'une des banques, l'entreprise a la possibilité de trouver un financement auprès des autres banques informées, qui ne craignent pas la «malédiction du vainqueur».

Dans quel cas est-il alors intéressant pour une entreprise de disposer d'une banque principale unique? Au-delà des raisons qui ne sont pas liées à la qualité de l'entreprise, et que nous évoquons ci-dessous, une entreprise peut avoir intérêt à ne pas se soumettre au contrôle de plusieurs banques. En effet, l'avantage informationnel réel ou supposé de la banque principale peut l'inciter à relâcher sa vigilance. Une relation de clientèle entre une banque et une entreprise repose sur une confiance à même de limiter la vigilance du créancier (Udell [1989]). Dès lors, une entreprise de qualité médiocre ou qui anticipe une dégradation de son résultat n'a pas intérêt à se soumettre à un contrôle double, voire triple. Les entreprises dont la performance économique est bonne ont intérêt à avoir plusieurs banques principales, alors que les entreprises de moindre qualité ont intérêt à nouer une relation exclusive. C'est la seconde hypothèse que nous désirons tester.

HYPOTHÈSE $\mathrm{n}^{\circ} 2$. Une entreprise de mauvaise qualité ou anticipant une dégradation de sa qualité se caractérise par un nombre de banques principales égal à un.

\section{Autres facteurs explicatifs du nombre de banques et de banques principales}

Parallèlement à la qualité de l'entreprise, d'autres facteurs sont susceptibles d'influencer son choix en termes de nombre de banques et de banques principales. En premier lieu, la taille et le niveau d'endettement de l'entreprise peuvent intervenir. La première raison en est le partage du risque entre banques : plus l'ampleur des financements externes est forte, plus l'entreprise a, a priori, besoin de recourir à plusieurs banques. De plus, il semble légitime de supposer que plus la taille et l'endettement sont élevés, et plus l'entreprise peut facilement mettre en place une stratégie de banques multiples ${ }^{1}$. Cela lui permettrait de plus de nouer plus aisément des relations privilégiées avec plusieurs banques : chaque banque reste incitée à investir informationnellement puisqu'elle prête pour un montant élevé. De même, un montant de dettes fournisseurs faible pourrait auto-

1. Pour une étude sur le lien entre nombre de banques et taille de l'entreprise, voir notamment Lefilliatre [2003]. 
riser plus facilement une stratégie de banques multiples et de banques principales multiples.

D'autres facteurs sont susceptibles d'intervenir dans la détermination du nombre de banques de l'entreprise. Tout d'abord une activité de recherche et développement intense peut limiter le nombre de banques principales pour des raisons de confidentialité. Au contraire, l'entreprise peut tenter de diluer l'information en possédant des sources de financement peu concentrées. L'activité de R\&D peut donc avoir un impact positif sur le nombre de banques. Enfin, l'émission d'obligations joue un rôle important dans la production d'informations. En réduisant l'asymétrie d'information, l'émission d'obligations réduit l'avantage informationnel d'une banque principale et diminue donc l'incitation d'une entreprise à nouer des relations privilégiées avec plusieurs banques. De même, l'émission d'obligations devrait réduire l'incitation d'une entreprise à diluer l'information par un financement bancaire diffus et donc diminuer le nombre total de banques.

\section{LES DONNÉES}

\section{Présentation générale des entreprises}

Tableau 1. Présentation de l'échantillon d'entreprises

\begin{tabular}{|c|c|c|c|c|c|c|c|c|}
\hline & $\begin{array}{c}\text { Chiffre } \\
\text { d'affaires (*) }\end{array}$ & $\begin{array}{l}\text { ETE/Capital } \\
\text { engagé }(\%)\end{array}$ & $\begin{array}{r}\text { Nom } \\
\text { ban }\end{array}$ & $\begin{array}{l}\text { bre de } \\
\text { ques }\end{array}$ & $\begin{array}{l}\text { Mon } \\
\text { crédit }\end{array}$ & $\begin{array}{l}\text { ant des } \\
\text { octroyés } \\
*)\end{array}$ & $\begin{array}{c}\text { Part de } \\
\text { chaque } \\
\text { banque }(\%)\end{array}$ & $\begin{array}{c}\text { Montant } \\
\text { octroyé par } \\
\text { banque }(*)\end{array}$ \\
\hline & & & Par an & Période & Par an & Période & & \\
\hline Moyenne & 117,17 & 9,39 & 3,56 & 5,42 & 5,05 & 25,23 & 18,46 & 4,66 \\
\hline $\begin{array}{c}\text { Variance } \\
\text { totale }\end{array}$ & 50275,75 & 104,70 & 10,71 & 20,26 & 165,48 & 3823,10 & 505,54 & 135,19 \\
\hline $\begin{array}{l}\text { Variance } \\
\text { Between }\end{array}$ & 48869,36 & 35,96 & 9,81 & - & 152,92 & - & - & - \\
\hline $\begin{array}{l}\text { Variance } \\
\text { Within }^{1}\end{array}$ & 1406,39 & 68,74 & 0,90 & - & 12,56 & - & - & - \\
\hline Minimum & 3,19 & $-55,28$ & 0 & 1 & 0 & 0,02 & 0,01 & 0,002 \\
\hline $\begin{array}{l}\text { Premier } \\
\text { quartile }\end{array}$ & 24,82 & 3,61 & 1 & 2 & 0,43 & 2,51 & 3,03 & 0,56 \\
\hline Médiane & 55,55 & 8,96 & 3 & 4 & 1,63 & 8,78 & 10,08 & 1,86 \\
\hline $\begin{array}{r}\text { Troisième } \\
\text { quartile }\end{array}$ & 125,51 & 14,73 & 5 & 7 & 4,90 & 24,20 & 24,65 & 4,89 \\
\hline Maximum & 3228,72 & 83,26 & 34 & 46 & 214,70 & 823,62 & 100 & 332,82 \\
\hline
\end{tabular}

(*) En millions de francs.

1. La variance within est la moyenne des variances individuelles alors que la variance between est la variance des moyennes individuelles. 
Nous utilisons des données individuelles de 565 firmes industrielles françaises - plus précisément de firmes du secteur des biens intermédiaires - fournies par la Banque de France. Nous nous limitons à un échantillon de PME, entreprises pour lesquelles la question de l'asymétrie d'information se pose avec le plus d'acuité. Pour chaque entreprise, nous disposons des comptes annuels issus des déclarations fiscales (données de la Centrale des bilans) et des informations relatives à l'octroi de prêts bancaires (données de la Centrale des risques). Les données sont disponibles sur la période 1993-1997.

Afin de décrire succinctement les caractéristiques générales de la population globale sur l'ensemble de la période, nous présentons, dans le tableau 1, la taille, représentée par le chiffre d'affaires et la profitabilité économique (décrite par le ratio Excédent de trésorerie d'exploitation sur capital engagé). L'échantillon se caractérise par une certaine disparité en termes de taille puisqu'il est constitué de moyennes, mais également de petites et de très petites entreprises. La profitabilité des entreprises est également hétérogène. Rares sont les entreprises qui présentent un ETE négatif, et $50 \%$ des entreprises ont un ratio ETE/capital engagé supérieur à $9 \%$.

\section{La structure de l'endettement bancaire des entreprises et leur multibancarité}

\section{Nombre de banques et ampleur du financement bancaire}

Il est tout d'abord intéressant de présenter les statistiques relatives au nombre de banques différentes auprès desquelles les entreprises se sont endettées par année mais également sur la période globale 1993-1997. Nous présentons également le montant total des crédits octroyés, par an et sur la période 1993-1997. Pour les données par année, nous calculons les variances within et between. La multibancarité est la stratégie largement majoritaire au sein de notre échantillon : seules 73 entreprises s'endettent qu' auprès d'une banque unique de 1993 à 1997. Le nombre total de banques sur la période varie de 1 à 46 , et ne varie que de 0 à 34 lorsqu' on considère le nombre de banques par année. Cependant, le troisième quartile est égal respectivement à 5 et $7:$ le nombre total de banques pour les PME de notre échantillon reste majoritairement inférieur à 10 . Une certaine disparité se retrouve également au niveau des montants globaux octroyés sur la période ou par année. Nous constatons que la disparité au sein de l'échantillon est moindre en termes de nombre de banques qu'en termes d'encours octroyé. La taille pourrait donc plus jouer sur le montant unitaire des crédits que sur le nombre de banques.

\section{Concentration du financement bancaire des entreprises}

Afin d'analyser la concentration du financement bancaire, nous présentons la part de chaque banque dans le financement bancaire global de l'entreprise de 1993 à 1997, ainsi que le montant octroyé par banque pendant les cinq années considérées. On peut constater une grande hétérogénéité quant au rôle joué par chaque banque dans le financement des entreprises, laissant supposer des stratégies différentes de la part des firmes. La part maximale du financement est évidemment $100 \%$, et sa valeur minimale est égale à $0,01 \%$. Le nombre de 
banques finançant à plus de $25 \%$ une entreprise particulière est limité. Comme précédemment, la disparité en termes de montant cumulé octroyé par banque est plus forte qu'en termes de part de chaque banque : la taille semble à nouveau plus influencer le montant du financement que sa structure.

\section{Présentation des variables retenues}

\section{Détermination des variables endogènes}

La première variable endogène que nous considérons est le nombre total de banques (BANQUE). Nous le définissons comme le nombre total de banques ayant octroyé un crédit à l'entreprise entre 1993 et 1997. Cette variable nous semble décrire fidèlement le nombre de banques avec lesquelles l'entreprise noue des relations financières.

La seconde variable endogène est le nombre de banques principales. La définition de ce qu'est une banque «principale » est délicat, particulièrement en France où les relations privilégiées ne sont pas aussi nettes qu'en Allemagne par exemple. De plus, il est difficile d'avoir accès à des informations telles que l'âge de la relation, l'implication éventuelle d'une banque dans l'actionnariat de l'entreprise etc. Grâce aux données de la Centrale des risques, nous pouvons néanmoins définir une banque principale par son poids dans le financement total de l'entreprise. Définir une norme est alors nécessaire. Comme précédemment, nous envisageons les montants globaux sur la période 1993-1997. Si l'entreprise a des relations avec une banque unique, celle-ci est par définition une banque principale. Si l'entreprise a des relations avec deux banques, nous assimilons une relation privilégiée unique au fait d'avoir une banque qui participe à plus de $70 \%$ dans le financement total de l'entreprise. L'autre participe alors à moins de $30 \%$. Notre norme revient donc à dire qu'une banque est principale dès qu'elle représente plus de $30 \%$ du financement bancaire total de l'entreprise sur la période 1993-1997. Si l'entreprise a des relations avec trois banques, nous réduisons ce seuil à $25 \%$. Enfin, si l'entreprise emprunte auprès de plus de trois banques, le seuil est fixé à $20 \%{ }^{1}$. Conformément à l'hypothèse 2 , nous choisissons comme variable expliquée une variable dichotomique (BQPR) : le fait de nouer une relation privilégiée unique (BQPR vaut 0 ) versus le fait de disposer de plusieurs banques principales (BQPR vaut 1). Parmi les 565 entreprises de l'échantillon initial, 239 se caractérisent par une relation de banque principale unique, 307 par plusieurs relations de banques principales et enfin seules 19 entreprises ne disposent d'aucune banque principale au sens où nous l'avons défini.

\section{Variables exogènes sélectionnées}

Les variables exogènes sont tirées des bilans et comptes de résultat des entreprises considérées. Puisque nous envisageons le nombre de banques et le nombre de banques principales sur la période 1993-1997, les variables explicatives sont majoritairement des moyennes de variables annuelles sur la période. Nous mesurons dans un premier temps la taille - par le logarithme du chiffre d'affaires -

1. Les résultats trouvés sont robustes aux seuils choisis. 
l'endettement financier total rapporté au capital engagé, les dettes fournisseurs rapportées à l'endettement financier et les dépenses en R\&D, que nous rapportons au chiffre d'affaires. Nous prenons également en compte l'émission d'obligations grâce à une variable dummy, qui vaut 1 si l'entreprise a réalisé une émission entre 1993 et 1997.

Conformément aux hypothèses que nous désirons tester, nous devons spécifier ce que les modèles théoriques désignent comme la «qualité » d'une entreprise. La «qualité » peut s'interpréter comme l'aptitude d'une entreprise à rembourser sa dette. Nous considérons donc en premier lieu la performance économique de l'entreprise : la rentabilité économique moyenne, ainsi que la rentabilité économique de fin de période, et le taux de croissance de la rentabilité sur la période de manière à intégrer les anticipations éventuelles des entreprises. Afin de limiter les problèmes de multicolinéarité, nous optons pour deux mesures différentes. L'une ramène l'EBE au capital engagé, l'autre ramène l'Excédent de trésorerie d'exploitation au capital engagé. D'autres variables sont nécessaires pour appréhender la «qualité » d'une entreprise. La liquidité de l'entreprise doit être prise en compte. Nous avons retenu le ratio standard Excédent brut d'exploitation rapporté aux frais financiers. Le risque de l'activité est également important. Nous le mesurons par la volatilité du chiffre d'affaires sur la période 1993-1997. Enfin, nous tenons compte de l'investissement, afin de refléter les potentialités de croissance de l'entreprise : nous considérons, d'une part, les investissements matériels déjà réalisés, donc l'équipement productif tel qu'il apparaît à l'actif, d'autre part, la rentabilité apparente de ces investissements.

Nous retenons finalement douze variables explicatives, présentées dans le tableau 2.

Tableau 2. Liste des variables explicatives

\begin{tabular}{l|l|c|c}
\hline & \multicolumn{1}{|c|}{ Définition } & $\begin{array}{c}\text { Impact attendu } \\
\text { sur BANQUE }\end{array}$ & $\begin{array}{c}\text { Impact attendu } \\
\text { sur BQPR }\end{array}$ \\
\hline TAILLE & ln(chiffre d'affaires) & + & + \\
\hline LEVIER & endettement financier total/capital engagé & + & + \\
\hline FOURN & dettes fournisseurs/endettement financier & - & - \\
\hline RD & dépenses en R\&D/chiffre d'affaires & + & - \\
\hline OBLIG & émission d'obligations (dummy) & - & + \\
\hline PROFIT & EBE/capital engagé & - & + \\
\hline PROFIT97 & ETE/capital engagé en 1997 & - & + \\
\hline CRPROFIT & taux de croissance de (ETE/capital engagé) & + & - \\
\hline LIQUID & EBE/frais financiers & - & + \\
\hline RISQUE & $\begin{array}{l}\text { écart type du taux de croissance du chiffre } \\
\text { d'affaires }\end{array}$ & - & + \\
\hline INV & équipement productif/capital engagé & - & + \\
\hline RINV & valeur ajoutée/équipement productif & - & +
\end{tabular}

Les variables sont des moyennes sur la période 1993-1997, sauf PROFIT97 et CRPROFIT. 


\section{ÉTUDE EMPIRIQUE}

\section{La méthodologie économétrique retenue}

Le nombre de banques total auprès desquelles les entreprises s'endettent entre 1993 et 1997 varie entre 1 et 46. Aussi utilisons-nous une régression par les moindres carrés ordinaires. Pour étudier le nombre de banques principales, nous utilisons un modèle Probit. La variable endogène que nous considérons est en effet une variable dichotomique. Nous la fixons égale à 0 lorsque l'entreprise noue une relation bancaire privilégiée unique et égale à 1 si elle noue plusieurs relations bancaires privilégiées. Nous estimons le vecteur des paramètres par la méthode du maximum de vraisemblance. La qualité de l'ajustement du modèle ainsi que la significativité de chaque paramètre estimé sont testées par le rapport de vraisemblance.

\section{Résultats}

Le tableau 3 présente les résultats des deux estimations, ainsi que la probabilité de se tromper en considérant que les coefficients estimés sont significativement différents de zéro. Les deux relations testées sont globalement significatives. La taille joue un rôle important au sein des deux régressions : elle a un impact positif sur le nombre de banques comme sur la probabilité qu'une entreprise noue plusieurs relations privilégiées. Le niveau d'endettement influence positivement le nombre de banques, conformément au résultat attendu. En revanche, il n'explique pas significativement le fait de disposer de plusieurs banques principales. La stratégie de disposer de plusieurs relations privilégiées n'est donc pas accentuée par un endettement plus élevé. Néanmoins, les dettes fournisseurs rapportées à l'endettement financier présentent un coefficient négatif dans la deuxième relation testée, ce qui tend à montrer que les entreprises peu endettées vis-à-vis de leur fournisseurs peuvent plus aisément mettre en place une stratégie de banque principale multiple. La relation est en revanche significative et positive en ce qui concerne le nombre de banques total. Une explication aurait pu se trouver dans le lien entre les difficultés d'une entreprise et son recours au crédit fournisseurs, mais aucune relation statistique de cet ordre n'a pu être observée au sein de l'échantillon.

Les dépenses en recherche et développement n'interviennent de manière significative dans aucune des relations testées. Ceci va à l'encontre de l'idée d'un désir accru de confidentialité en cas d'activité de recherche importante. Par ailleurs, le fait d'émettre des obligations réduit la probabilité que l'entreprise ait plusieurs banques principales. L'idée selon laquelle cette stratégie est motivée par la volonté d'échapper au monopole induit par une relation exclusive est donc corroborée. L'émission d'obligations a cependant un impact positif sur le nombre total de banques. Ceci peut sans doute s'expliquer par le fait que les banques acceptent de prêter aux entreprises qui émettent des obligations, même si le montant des crédits octroyés ne permet pas d'investissement informationnel. 
Tableau 3. Résultats des régressions

\begin{tabular}{|c|c|c|c|c|}
\hline & \multicolumn{2}{|c|}{ Nombre total de banques } & \multicolumn{2}{|c|}{ Relation de banque principale } \\
\hline & Coefficient estimé & Probabilité & Coefficient estimé & Probabilité \\
\hline Constante & $-16,941$ & $0 \%$ & $-2,283$ & $0 \%$ \\
\hline TAILLE & 1,829 & $0 \%$ & 0,235 & $0 \%$ \\
\hline LEVIER & 16,263 & $0 \%$ & n.s. & n.s. \\
\hline FOURN & 0,019 & $0,4 \%$ & $-0,041$ & $0 \%$ \\
\hline $\mathrm{RD}$ & n.s. & n.s. & n.s. & n.s. \\
\hline OBLIG & 3,322 & $0 \%$ & $-1,002$ & $0 \%$ \\
\hline PROFIT & $-4,521$ & $10,7 \%$ & n.s. & n.s. \\
\hline PROFIT97 & 4,833 & $0,2 \%$ & n.s. & n.s. \\
\hline CRPROFIT & $-0,014$ & $6,1 \%$ & n.s. & n.s. \\
\hline LIQUID & $-0,002$ & $9,2 \%$ & $-0,004$ & $0,3 \%$ \\
\hline RISQUE & n.s. & n.s. & 0,953 & $6,7 \%$ \\
\hline INV & n.s. & n.s. & n.s. & n.s. \\
\hline RINV & $-1,154$ & $0 \%$ & n.s. & n.s. \\
\hline & $\mathrm{R}^{2}$ & probabilité & Log-vraisemblance & probabilité \\
\hline Modèle & $54,5 \%$ & $0 \%$ & $-343,814$ & $0 \%$ \\
\hline
\end{tabular}

Enfin, les conclusions que nous pouvons tirer quant au rôle joué par la qualité de l'entreprise sur le nombre de banques tendent à corroborer notre hypothèse 1 . Quatre variables influencent significativement le nombre total de banques dans le sens attendu : la liquidité, la rentabilité économique moyenne, son taux de croissance sur la période et la rentabilité apparente des investissements. Il semblerait donc qu'un nombre élevé de banques s'accompagne effectivement d'une moindre performance de l'entreprise, et même d'une détérioration anticipée par la firme de sa qualité. Néanmoins, la rentabilité économique de fin de période, elle, a un impact positif sur le nombre de banques. De surcroît, l'investissement et le risque ne jouent pas de rôle significatif.

Concernant le nombre de banques principales, aucune conclusion marquée n'apparaît relativement à l'hypothèse 2 : seuls la liquidité et le risque d'exploitation ont une influence significative sur la probabilité que l'entreprise ait une relation de banque principale multiple. La liquidité a un signe négatif, et le risque un signe positif, ce qui va à l'encontre de l'hypothèse 2 . Bénéficier de plusieurs banques principales ne semble pas une stratégie recherchée par les entreprises liquides et stables et donc peu soumises au risque de défaut. Au contraire, une entreprise pourrait tenter de disposer de plusieurs banques principales en cas de difficultés financières anticipées, espérant peut-être un soutien financier plus aisé.

\section{CONCLUSION}

À partir de données comptables d'entreprises industrielles françaises et de données relatives aux crédits dont elles ont bénéficié, nous avons voulu mettre en évidence le lien entre la qualité des entreprises et leur stratégie de multiban- 
carité. Selon les résultats mis en exergue par la littérature théorique, choisir un nombre global de banques élevé permet aux entreprises médiocres d'échapper à la surveillance trop étroite d'une banque principale; nouer avec plusieurs banques des relations privilégiées permet aux entreprises performantes de ne pas pâtir de l'exploitation par une banque principale unique de sa rente informationnelle. Les résultats trouvés tendent à montrer en effet que le nombre de banques décroît avec la qualité de l'entreprise, si on la mesure par la rentabilité économique de l'entreprise, par le taux de croissance de cette rentabilité, par sa liquidité et par la performance apparente de ses investissements matériels. Contrairement à l'hypothèse théorique testée, la volonté de l'entreprise de nouer une relation privilégiée unique n'est que peu liée significativement avec sa qualité. Seuls le risque d'exploitation et une liquidité faible accroissent la probabilité de disposer de plusieurs banques principales, laissant donc supposer un lien négatif entre la qualité de l'entreprise et une relation de banques principales multiples. Néanmoins, le rôle joué par l'émission d'obligations, liée positivement au fait de disposer d'une banque principale unique, corrobore l'idée qu'une stratégie de banques principales multiples est motivée par des raisons informationnelles.

\section{RÉFÉRENCES BIBLIOGRAPHIQUES}

ACTUALITÉ BANCAIRE [2001], « La banque en ligne ne modifie pas la relation à l'agence et le passage à l'euro des PME est encore loin d'être réalisé », $n^{\circ} 447$, janvier.

AgARWAL R., ELSTON J. [2001], «Bank-firm relationships, financing and firm performance in Germany », Economics Letters, 72, p. 225-232.

BERgER A., KLAPPER L., UdELl G. [2001], « The ability of banks to lend to informationally opaque small businesses », Journal of Banking and Finance, 25 (12), p. 2127 2167.

CARLETTI A. [2002], « The structure of bank relationships, endogenous monitoring and loan rates », Document de travail, Université de Mannheim, janvier.

D'Auria C., Foglia A. et ReEdTZ P. M. [1999], « Bank interest rates and credit relationships in Italy », Journal of Banking and Finance, 23, p. 1067-1093.

De BodT E., LOBEZ F. et STATNIK J.-C. [2000], « Décision de crédit et risque moral de substitution d'actifs: une étude empirique des comportements bancaires ", $17^{\mathrm{e}}$ Journées internationales de finance, 26 et 27 juin 2000, Paris.

DE BodT E., LobeZ F. et STATNIK J.-C. [2001], « Credit rationing, customer relationship and the number of banks - an empirical analysis ", Document de travail.

Detragiache E., Garella P. et Guiso L. [2000], « Multiple versus Single Banking Relationships: Theory and Evidence », Journal of Finance, 55 (2), juin, p. 1133-1161.

DIAMOND D.W. [1984], «Financial intermediation and delegated monitoring », Review of Economic Studies, 51, p. 393-414.

FAMA E.F. [1985], «What's different about banks? », Journal of Monetary Economics, 15, p. 29-39.

Foglia A., LAVIOLA S. et MARULlo ReEDTZ P. [1998], « Multiple banking relationships and the fragility of corporate borrowers », Journal of Banking and Finance, 22 (1011), p. 1441-1456.

Gangopadhyay S., Mukhopadhyay B. [2002], « Multiple bank lending and seniority in claims », Journal of Economics And Business, 54 (1), p. 7-30.

LEFILLIATRE D. [2003], «La multibancarité », Cahier d'études et recherches de l'Observatoire des entreprises, Banque de France. 
OngENA S. et SMITH D. [2000], «What determines the number of bank relationships? Cross-country evidence », Journal of Financial Intermediation, 9, p. 26-56.

ONGENA S. et SMITH D. [2001], « The duration of bank relationships », Journal of Financial Economics, 61, p. 449-475.

RAJAN R.G. [1992], «Insiders and Outsiders: The Choice between Informed and Arm's length Debt », Journal of Finance, 47 (4), septembre, p. 1367-1400

SHARPE S.A. [1990], « Asymmetric information, bank lending and implicit contracts: A stylized model of customer relationships », Journal of Finance, 45 (4), septembre, p. 1069-1087.

UDELL G.F. [1989], « Loan quality, commercial loan review and loan officer contracting », Journal of Banking and Finance, 13, p. 367-382.

VAUBOURG A.-G. [2000], «Essais sur l'architecture de systèmes financiers : analyse théorique et empirique du partage entre banque et marché », Thèse pour le doctorat en sciences économiques, Université d'Orléans, novembre.

VON THADEN E.L. [1994], « The commitment in finance, duplicated monitoring and the investment horizon ", Unpublished manuscript, Basel.

WinTon A. [1995], "Costly state verification and multiple investors: the role of seniority », Review of Financial Studies, 8, p. 91-123. 